CREAT. MATH. INFORM.

Volume 25 (2016), No. 2,

Pages 237 - 247
Online version at https : //creative-mathematics. cunbm. utcluj.ro/

Print Edition: ISSN 1584 - 286X; Online Edition: ISSN 1843 - 441X

DOI: https://doi.org/10.37193/CMI.2016.02.15

\title{
On orthonormal sets in inner product quasilinear spaces
}

\author{
YILMAZ YILMAZ ${ }^{2}$, HACER BOZKURT ${ }^{1}$ and SÜMEYYE ÇAKAN ${ }^{2}$
}

\begin{abstract}
Aseev, S. M [Aseev, S. M., Quasilinear operators and their application in the theory of multivalued mappings, Proc. Steklov Inst. Math., 2 (1986), 23-52] generalized linear spaces by introducing the notion of quasilinear spaces in 1986. Then, special quasilinear spaces which are called "solid floored quasilinear spaces" were defined and their some properties examined in [ Çakan, S., Some New Results Related to Theory of Normed Quasilinear Spaces, Ph.D. Thesis, İnönü University, Malatya, 2016]. In fact, this classification was made so as to examine consistent and detailed some properties related to quasilinear spaces. In this paper, we present some properties of orthogonal and orthonormal sets on inner product quasilinear spaces. At the same time, the mentioned classification is crucial for define some topics such as Schauder basis, complete orthonormal sequence, orthonormal basis and complete set and some related theorems. Also, we try to explain some geometric differences of inner product quasilinear spaces from the inner product (linear) spaces.
\end{abstract}

\section{INTRODUCTION}

In 1986, Aseev generalized linear spaces by introducing the notion of quasilinear spaces, briefly, QLSs. He used the partial order relation to get an extended definition and hence can give consistent counterparts of some fundamental results of linear algebra. For more details, reader can refer to [1]. After this work, a lot of authors have motivated to introduce new results on set-valued analysis [2], set-differential equations [6], fuzzy quasilinear spaces [7], etc.

Inspired and motivated by research going on this area, we introduce concept of inner product quasilinear spaces, IPQLS for short. We give also the concept of orthogonality in this cite and present some informations about the geometry of quasilinear space. Then, we realized that geometry in quasilinear spaces may be different from the geometry of linear spaces with some aspects.

Let us continue by giving the definition of quasilinear space and some its basic properties given by Aseev [1].

Definition 1.1. A set $X$ is called a quasilinear space (QLS, for short) if a partial order relation " $\preceq$ ", an algebraic sum operation, and an operation of multiplication by real numbers are defined in it in such a way that the following conditions hold for any elements $x, y, z, v \in X$ and any $\alpha, \beta \in \mathbb{R}$ :

(1) $x \preceq x$,

(2) $x \preceq z$ if $x \preceq y$ and $y \preceq z$,

(3) $x=y$ if $x \preceq y$ and $y \preceq x$,

(4) $x+y=y+x$

(5) $x+(y+z)=(x+y)+z$,

(6) there exists an element $\theta \in X$ such that $x+\theta=x$,

(7) $\alpha \cdot(\beta \cdot x)=(\alpha \cdot \beta) \cdot x$,

Received: 28.01.2016. In revised form: 08.05.2016. Accepted: 26.05.2016

2010 Mathematics Subject Classification. 46C05, 46C07, 46C15, 46C50, 97H50.

Key words and phrases. Quasilinear space, Inner product quasilinear space, Hilbert quasilinear space, Orthogonality, Orthonormality.

Corresponding author: Hacer Bozkurt; hacerbozkurt86@gmail.com 
(8) $\alpha \cdot(x+y)=\alpha \cdot x+\alpha \cdot y$,

(9) $1 \cdot x=x$,

(10) $0 \cdot x=\theta$,

(11) $(\alpha+\beta) \cdot x \preceq \alpha \cdot x+\beta \cdot x$,

(12) $x+z \preceq y+v$ if $x \preceq y$ and $z \preceq v$,

(13) $\alpha \cdot x \preceq \alpha \cdot y$ if $x \preceq y$.

A linear space is a QLS with the partial order relation "=".

The most popular example which is not a linear space is the set of all closed intervals of real numbers with the inclusion relation " $\subseteq$ ", the algebraic sum operation

$$
A+B=\{a+b: a \in A, b \in B\}
$$

and the real-scalar multiplication

$$
\lambda \cdot A=\{\lambda a: a \in A\} .
$$

We denote this set by $\Omega_{C}(\mathbb{R})$.

Another one is $\Omega(\mathbb{R})$, which is the set of all compact subsets of real numbers. By a slight modification of algebraic sum operation (with closure) such as

$$
A+B=\overline{\{a+b: a \in A, b \in B\}},
$$

with the same real-scalar multiplication defined above and the inclusion relation we can give as example the nonlinear QLS $\Omega(E)$ and $\Omega_{C}(E)$, the families of all nonempty closed bounded and convex closed bounded subsets of the normed linear space $E$, respectively.

Lemma 1.1. Suppose that every element $x$ in a QLS $X$ has an inverse element $x^{\prime} \in X$. Then the partial order relation in $X$ is determined by equality, the distributivity condition in (11) holds, and consequently, $X$ is a linear space [1].

Let $X$ be a QLS and $Y \subseteq X$. Then $Y$ is called a subspace of $X$ whenever $Y$ is a QLS with the same partial order and the restriction to $Y$ of the operations on $X$. One can easily prove the following theorem by using the axioms of to be a QLS. It is quite similar to its linear space analogue.

Theorem 1.1. [8] Let $X$ be a QLS and $Y \subseteq X$. Then $Y$ is a subspace of $X$ if and only if $\alpha \cdot x+$ $\beta \cdot y \in Y$ for every, $x, y \in Y$ and $\alpha, \beta \in \mathbb{R}$.

Let $X$ be a QLS. An element $x \in X$ is said to be symmetric if $-x=x$, where $-x=$ $(-1) \cdot x$, and $X_{d}$ denotes the set of all symmetric elements. $\theta$ denotes the unit element of addition operation in $X$, and it is minimal element, i.e., $x=\theta$ if $x \preceq \theta$. An element $x^{\prime}$ is called inverse of $x$ if $x+x^{\prime}=\theta$. If the inverse element exists, then it is unique and $x^{\prime}=-x$ in this case. Sometimes $x^{\prime}$ may not be exist but $-x$ is always meaningful in quasilinear spaces. An element $x$ possessing inverse is called regular, otherwise is called singular. Now, $X_{r}$ and $X_{s}$ stand for the sets of all regular and singular elements in $X$, respectively. Further, $X_{r}, X_{d}$ and $X_{s} \cup\{0\}$ are subspaces of $X$ and they are called regular, symmetric and singular subspaces of $X$, respectively [8]. It is easy from the definitions that $X_{d} \subset X_{s}$ and $X=X_{r} \cup X_{s}$.

For a singular element $x$ we should note that $x-x \neq \theta$. In a linear QLS, that is, in a linear space, there are no singular elements. Also, in a QLS $X$, it is obvious that an element $x$ is regular if and only if $x-x=\theta$.

Proposition 1.1. [4] Let $X$ be a $Q L S X, x \in X_{r}$ and $y \in X_{s}$ then $x+y \in X_{s}$.

Proof. Suppose that $x+y \in X_{r}$. By the definitions

$$
(x+y)-(x+y)=(x-x)-(y-y)=y-y=\theta .
$$


Hence $y \in X_{r}$. This contradicts with $y \in X_{s}$.

Proposition 1.2. [8] In a quasilinear space $X$ every regular element is minimal.

Definition 1.2. Let $X$ be a QLS. A function $\|\cdot\|_{X}: X \longrightarrow \mathbb{R}$ is called a norm if the following conditions satisfied:

(14) $\|x\|_{X}>0$ if $x \neq \theta$,

(15) $\|x+y\|_{X} \leq\|x\|_{X}+\|y\|_{X}$,

(16) $\|\alpha \cdot x\|_{X}=|\alpha|\|x\|_{X}$,

(17) if $x \preceq y$, then $\|x\|_{X} \leq\|y\|_{X}$,

(18) if for any $\varepsilon>0$ there exists an element $x_{\varepsilon} \in X$ such that $x \preceq y+x_{\varepsilon}$ and $\left\|x_{\varepsilon}\right\|_{X} \leq \varepsilon$ then $x \preceq y$.

A QLS $X$ with a norm defined on it, is called normed quasilinear space (briefly, normed $Q L S)$.

It follows from Lemma 1.1 that if every $x \in X$ has inverse element $x^{\prime} \in X$, then the concept of normed QLS coincides with the concept of normed linear space, [1].

Let $X$ be a normed QLS. Hausdorff metric or norm metric on $X$ is defined by the equality

$$
h_{X}(x, y)=\inf \left\{r \geqslant 0: x \preceq y+a_{1}^{r}, y \preceq x+a_{2}^{r} \text { and }\left\|a_{i}^{r}\right\| \leq r, i=1,2\right\} .
$$

Since $x \preceq y+(x-y)$ and $y \preceq x+(y-x)$, the quantity $h_{X}(x, y)$ is well-defined and satisfies all of the metric axioms, also

$$
h_{X}(x, y) \leq\|x-y\|_{X}
$$

for all elements $x, y \in X$.

Lemma 1.2. [1] The operations of algebraic sum and multiplication by real numbers are continuous with respect to the Hausdorff metric. The norm is continuous function respect to the Hausdorff metric.

Example 1.1. Let $E$ be a normed linear space. A norm on $\Omega(E)$ is defined by

$$
\|A\|_{\Omega(E)}=\sup _{a \in A}\|a\|_{E} .
$$

$\Omega_{C}(E)$ is a also a normed qls with the norm above. In this case, the Hausdorff metric on $\Omega(E)$ and $\Omega_{C}(E)$ is defined as usual:

$$
h_{\Omega}(A, B)=\inf \left\{r \geqslant 0: A \subset B+S_{r}(\theta), B \subset A+S_{r}(\theta)\right\},
$$

where $S_{r}(\theta)$ is the closed ball of radius $r$ and centered at $\theta \in X$, [1].

Definition 1.3. [3] Let $X$ be a quasilinear space, $\left\{x_{k}\right\}_{k=1}^{n} \subset X$. The element $x \in X$ with

$$
\alpha_{1} \cdot x_{1}+\alpha_{2} \cdot x_{2}+\ldots+\alpha_{n} \cdot x_{n} \preceq x
$$

is said to be a quasilinear combination (ql-combination, for short) of $\left\{x_{k}\right\}_{k=1}^{n}$ corresponding to scalars $\left\{\alpha_{k}\right\}_{k=1}^{n} \subset \mathbb{R}$.

The set

$$
Q s p A=\left\{x \in X: \sum_{k=1}^{n} \alpha_{k} \cdot x_{k} \preceq x, x_{1}, x_{2}, \ldots, x_{n} \in A, \alpha_{1}, \alpha_{2}, \ldots, \alpha_{n} \in \mathbb{R}\right\}
$$

is said to be quasispan of $A$, and is denoted by $Q s p A$. One can see easily that $Q s p A$ is a subspace of $X$. Ql-combinations of $\left\{x_{k}\right\}_{k=1}^{n}$ corresponding the scalars $\left\{\alpha_{k}\right\}_{k=1}^{n}$ may not be unique, from the definition, while it is well known that the linear combination of $\left\{x_{k}\right\}_{k=1}^{n}$ corresponding these scalars is unique.

It is clear that $S p A \subseteq Q \operatorname{sp} A$. If $X$ is a linear space then $Q \operatorname{sp} A=S p A$, where $S p A$ is span of $A \subseteq X$. 
Definition 1.4. [3] Let $X$ be a QLS, $\left\{x_{k}\right\}_{k=1}^{n} \subset X$ and $\left\{\lambda_{k}\right\}_{k=1}^{n} \subset \mathbb{R}$. If

$$
\theta \preceq \lambda_{1} \cdot x_{1}+\lambda_{2} \cdot x_{2}+\ldots+\lambda_{n} \cdot x_{n}
$$

implies $\lambda_{1}=\lambda_{2}=\ldots=\lambda_{n}=0$, then $\left\{x_{k}\right\}_{k=1}^{n}$ is said to be quasilinear independent (briefly, ql-independent), otherwise $\left\{x_{k}\right\}_{k=1}^{n}$ is said to be quasilinear dependent (ql-dependent, for short).

Definition 1.5. [5] Let $S$ be a ql-independent subset of the QLS $X . S$ is called maximal ql-independent subset of $X$ whenever $S$ is ql-independent, but any superset of $S$ is not qlindependent.

Definition 1.6. [5] Regular (Singular) dimension of any $Q L S X$ is the cardinality of maximal ql-independent subsets of $X_{r}\left(X_{s}\right)$. If this number is finite then $X$ is called finite regular (singular)-dimensional, otherwise; is called infinite regular (singular)-dimensional.

Regular and singular dimensions are denoted by $r-\operatorname{dim} X$ and $s$-dim $X$, respectively. If $r$ - $\operatorname{dim} X=a$ and $s$-dim $X=b$ then we say that $X$ is an $\left(a_{r}: b_{s}\right)$-dimensional QLS.

By the fact that the subspace $X_{r}$ of $X$ is a linear space we can also define as regular dimension of $X$ is dimension of linear space $X_{r}$. This also means regular dimension $X$ is the cardinality of any maximal linearly independent subsets of the linear space $X_{r}$. According to this, $r$ - $\operatorname{dim} X=\operatorname{dim} X_{r},[4]$.

From the definition we can easily see that any $n$-dimensional linear space is an $\left(n_{r}: 0_{s}\right)$ dimensional QLS. Then, the trivial linear space $\{0\}$ is a $\left(0_{r}: 0_{s}\right)$-dimensional QLS.

Remark 1.1. If we say a QLS $X$ is finite-dimensional, precisely, $n$-dimensional we mean an $\left(n_{r}: n_{s}\right)$ or $\left(n_{r}: 0_{s}\right)$-dimensional QLS. Especially, $\left(n_{r}: n_{s}\right)$-dimensional QLS is an $n$ dimensional nonlinear QLS while $\left(n_{r}: 0_{s}\right)$-dimensional QLS is an $n$-dimensional linear space.

Example 1.2. $\Omega(\mathbb{R})$ and $\Omega_{C}(\mathbb{R})$ are 1-dimensional nonlinear quasilinear spaces. But singular subspace of $\Omega_{C}(\mathbb{R})$ is $\left(0_{r}: 1_{s}\right)$-dimensional QLS. Further, $\mathbb{R}$ is $\left(1_{r}: 0_{s}\right)$-dimensional QLS, equivalently, 1-dimensional linear space. Especially, every linear space is a QLS and has $\left(n_{r}: 0_{s}\right)$ dimension, where $n$ is the dimension of the linear space and it may be infinite.

Definition 1.7. [3] Let $X$ be a QLS and $A$ be a ql-independent subset of $X$. If $Q \operatorname{sp} A=X$, then the set $A$ is called a (Hamel) basis for $X$.

Now, let us give a useful type of QLSs called solid-floored to give notions of (Schauder) basis, orthonormal basis and complete orthonormal basis in an IPQLS.

Definition 1.8. [5] Let $X$ be a qls, $M \subseteq X$ and $x \in M$. The set

$$
F_{x}^{M}=\left\{z \in M_{r}: z \preceq x\right\}
$$

is called floor in $M$ of $x$. In the case of $M=X$ it is called only floor of $x$ and written briefly $F_{x}$ instead of $F_{x}^{X}$.

Floor of an element $x$ in linear spaces is $\{x\}$. Therefore, it is nothing to discuss the notion of floor of an element in a linear space.

Definition 1.9. [5] Let $X$ be a qls and $M \subseteq X$. Then the union set

$$
\bigcup_{x \in M} F_{x}^{M}
$$

is called floor of $M$ and is denoted by $\mathcal{F}_{M}$. In the case of $M=X, \mathcal{F}_{X}$ is called floor of the qls $X$. 
On the other hand, the set

$$
\mathcal{F}_{M}^{X}=\bigcup_{x \in M} F_{x}^{X}
$$

is called floor in $X$ of $M$ and is denoted by $\mathcal{F}_{M}^{X}$.

We refer to the reader to [5] for detailed informations about this topic.

Definition 1.10. [4] A quasilinear space $X$ is called solid floored qls (sf-qls, for short) whenever $\sup F_{y}$ exists for every $y \in X$ and

$$
y=\sup F_{y}=\sup \left\{z \in X_{r}: z \preceq y\right\} .
$$

Otherwise, $X$ is called non-solid floored qls and in this case it is written as nsf-qls sake for brevity.

Especially, we should note that the supremum in this definition is considered according to relation " $\preceq$ " on the qls $X$.

Example 1.3. [4] For any normed linear space $E, \Omega(E)$ and $\Omega_{C}(E)$ are sf-NQLS.

On the other hand, it is clear that $\left(\Omega_{C}(\mathbb{R})\right)_{s} \cup\{0\}$ is nsf-qls. For example,

$$
\sup \left\{x: x \in\left(\left(\Omega_{C}(\mathbb{R})\right)_{s} \cup\{0\}\right)_{r}, x \subseteq y\right\}=\{0\} \neq y
$$

for element $y=[-2,3] \in\left(\Omega_{C}(\mathbb{R})\right)_{s} \cup\{0\}$. Also, no there exists any element $x$ such that $x \subseteq z$ for $z=[1,3] \in\left(\Omega_{C}(\mathbb{R})\right)_{s} \cup\{0\}$.

\section{INNER PRODUCT QUASILINEAR SPACES}

Before giving the definition of inner product quasilinear spaces, we must give following definition.

Definition 2.11. Let $X$ be a QLS. Consolidation of floor of $X$ is the smallest solid-floored QLS $\widehat{X}$ containing $X$, that is, if there exists another solid-floored QLS $Y$ containing $X$ then $\widehat{X} \subseteq Y$.

Clearly, $\widehat{X}=X$ for some solid-floored QLS $X$. Further, $\widehat{\Omega_{C}\left(\mathbb{R}^{n}\right)_{s}}=\Omega_{C}\left(\mathbb{R}^{n}\right)$. For a QLS $X$, the set

$$
F_{y}^{\widehat{X}}=\left\{z \in(\widehat{X})_{r}: z \preceq y\right\} .
$$

is the floor of $X$ in $\widehat{X}$.

We can say that the inner poduct in the following definition may be seen a set-valued inner product on quasilinear spaces.

Definition 2.12. Let $X$ be a quasilinear space. A mapping $\langle\rangle:, X \times X \rightarrow \Omega(\mathbb{R})$ is called an inner product on $X$ if the following conditions are satisfied for any $x, y, z \in X$ and $\alpha \in \mathbb{R}$ :

$(19)$ if $x, y \in X_{r}$ then $\langle x, y\rangle \in\left(\Omega_{C}(\mathbb{R})\right)_{r} \equiv \mathbb{R}$,

(20) $\langle x+y, z\rangle \subseteq\langle x, z\rangle+\langle y, z\rangle$,

(21) $\langle\alpha \cdot x, y\rangle=\alpha \cdot\langle x, y\rangle$,

(22) $\langle x, y\rangle=\langle y, x\rangle$,

(23) $\langle x, x\rangle \geq 0$ for $x \in X_{r}$ and $\langle x, x\rangle=0 \Leftrightarrow x=\theta$,

(24) $\|\langle x, y\rangle\|_{\Omega(\mathbb{R})}=\sup \left\{\|\langle a, b\rangle\|_{\Omega(\mathbb{R})}: a \in F_{x}^{\widehat{X}}, b \in F_{y}^{\widehat{X}}\right\}$,

(25) if $x \preceq y$ and $u \preceq v$ then $\langle x, u\rangle \subseteq\langle y, v\rangle$,

(26) if for any $\varepsilon>0$ there exists an element $x_{\varepsilon} \in X$ such that $x \preceq y+x_{\varepsilon}$ and $\left\langle x_{\varepsilon}, x_{\varepsilon}\right\rangle \subseteq$ $S_{\varepsilon}(\theta)$ then $x \preceq y$.

A quasilinear space with an inner product is called inner product quasilinear space, briefly, IPQLS. 
Example 2.4. One can easily seen that $\Omega_{C}(\mathbb{R})$, the space of closed real intervals, is a IPQLS with inner-product defined by

$$
\langle A, B\rangle=\{a \cdot b: a \in A, b \in B\} .
$$

Every IPQLS $X$ is a normed QLS with the norm defined by

$$
\|x\|=\sqrt{\|\langle x, x\rangle\|_{\Omega(\mathbb{R})}}
$$

for every $x \in X$. This norm is called inner-product norm. The classical norm of $\Omega_{C}(\mathbb{R})$ is generated by the above inner-product.

Proposition 2.3. $x_{n} \rightarrow x$ and $y_{n} \rightarrow y$ in an IPQLS then $\left\langle x_{n}, y_{n}\right\rangle \rightarrow\langle x, y\rangle$.

An IPQLS is called Hilbert QLS, if it is complete according to the inner-product (norm) metric. $\Omega_{C}(\mathbb{R})$ is a Hilbert QLS.

Definition 2.13. (Orthogonality) The element $x$ of an IPQLS $X$ is said to be orthogonal to the an element $y \in X$ if

$$
\|\langle x, y\rangle\|_{\Omega(\mathbb{R})}=0 .
$$

We say also that $x$ and $y$ are orthogonal and write $x \perp y$. Similarly, for subsets $A, B \subseteq X$ we write $x \perp A$ if $x \perp z$ for all $z \in A$ and $A \perp B$ if $a \perp b$ for all $a \in A$ and $b \in B$.

An orthonormal subset $M$ of $X$ is an orthogonal set in $X$ consisting elements have norm 1 , that is, for all $x, y \in M$

$$
\|\langle x, y\rangle\|_{\Omega(\mathbb{R})}=\left\{\begin{array}{c}
0, x \neq y \\
1, x=y
\end{array} .\right.
$$

Definition 2.14. Let $A$ be a nonempty subset of an IPQLS $X$. An element $x \in X$ is said to be orthogonal to $A$, denoted by $x \perp A$, if $\|\langle x, y\rangle\|_{\Omega(\mathbb{R})}=0$ for every $y \in A$. The set of all elements of $X$ orthogonal to $A$ which is denoted by $A^{\perp}$ is called the orthogonal complement of $A$ and is indicated by

$$
A^{\perp}=\left\{x \in X:\|\langle x, y\rangle\|_{\Omega(\mathbb{R})}=0, y \in A\right\} .
$$

For any subset $A$ of an IPQLS $X, A^{\perp}$ is a closed subspace of $X$.

\section{MAIN RESUlTS}

Definition 3.15. Let $X$ be a normed sf-QLS. A Schauder basis is a sequence $\left(x_{n}\right)$ of elements of $X$ such that for every element $y \in X$ and $z \in F_{y}$ there exists a unique sequence $\left\{\alpha_{n}^{(z)}\right\}_{n=1}^{\infty}$ of scalars such that

$$
z=\sum_{n=1}^{\infty} \alpha_{n}^{(z)} \cdot x_{n} \text { and } y=\sup \left\{z \in X_{r}: z \preceq y\right\} .
$$

As a result of this definition we can say that $\left(x_{n}\right)$ is a Schauder basis of $X$ iff

$$
h\left(z, \sum_{n=1}^{k} \alpha_{n}^{(z)} \cdot x_{n}\right) \rightarrow 0 \quad(k \rightarrow \infty) \text { and } y=\sup \left\{z \in X_{r}: z \preceq y\right\}
$$

for every $y \in X$ and $z \in F_{y}$.

Proposition 3.4. Let $X$ be a sf-QLS quasilinear space and $\left(x_{n}\right)$ be a Schauder basis of $X$. Then each $x_{n}, n \in \mathbb{N}$, must be regular. 
Proof. Since $X$ is a nontrivial sf-qls, there exists an element $y \neq \theta$ in $X_{r}$. Since $F_{y}=\{y\}$ and $\sup \left\{F_{y}\right\}=y$ for this element, there is a unique sequence of scalars $\left\{\alpha_{n}\right\}$ such that

$$
y=\sum_{n=1}^{\infty} \alpha_{n} \cdot x_{n} .
$$

Firstly; all terms of the sequence $\left\{x_{n}\right\}$ can not be zero. Because, in this case, it can not be written $y=\sum_{n=1}^{\infty} \alpha_{n} \cdot x_{n}$.

On the other hand, assume that some of terms $x_{n}$ be regular, e.g., it may be considered that $x_{1}$ is regular without los of generality. Then, there exists a regular element $u_{n}$ such that $u_{n} \preceq x_{n}$ for each $x_{n}, n \neq 1$. By the qls axioms (12) and (13), we write

$$
\sum_{n=2}^{\infty} \alpha_{n} \cdot u_{n} \preceq \sum_{n=2}^{\infty} \alpha_{n} \cdot x_{n}
$$

and then

$$
\alpha_{1} \cdot x_{1}+\sum_{n=2}^{\infty} \alpha_{n} \cdot u_{n} \preceq \sum_{n=1}^{\infty} \alpha_{n} \cdot x_{n} .
$$

Taking into account this result, the equality (3.1) and because of the fact that regular element $y$ is minimal; we obtain

$$
y=\sum_{n=1}^{\infty} \alpha_{n} \cdot x_{n}=\alpha_{1} \cdot x_{1}+\sum_{n=2}^{\infty} \alpha_{n} \cdot u_{n} .
$$

This implies $\sum_{n=2}^{\infty} \alpha_{n} \cdot u_{n}=\sum_{n=2}^{\infty} \alpha_{n} \cdot x_{n}$. Whereas; $\sum_{n=2}^{\infty} \alpha_{n} \cdot x_{n}$ and $\sum_{n=2}^{\infty} \alpha_{n} \cdot u_{n}$ are singular and regular elements, respectively. This contradiction completes the proof.

It is not hard to see the fact that if $\left(x_{n}\right)$ is a Hamel basis for a finite dimensional normed sf-QLS $X$, then $\left(x_{n}\right)$ is a Schauder basis for $X$.

Theorem 3.2. If a normed sf-QLS has a Schauder basis, then it is separable.

Proof. Let $\left\{x_{n}\right\}$ be a Schauder basis for a normed sf-QLS $X, M$ be a countable dense subset of $\mathbb{R}$, and $E_{n}$ be a set of all $z \in F y$ such that

$$
z=\sum_{i=1}^{n} m_{i}^{(z)} \cdot x_{i}, \quad m_{i} \in M,
$$

where $y$ is an element of $X$. Here, $m_{i}$ scalars determined uniquely by $z$. Therefore, there is a one to one relation between $E_{n}$ and $M$. So, $E_{n}$ is countable. If we say $E=\bigcup_{n=1}^{\infty} E_{n}$, then $E$ is a countable set. On the other hand, there is unique sequence $\left\{k_{n}\right\}$ in $\mathbb{R}$ determined by $z$ such that

$$
z=\sum_{n=1}^{\infty} k_{n} \cdot x_{n}
$$

for $z \in F_{y}$. Here, we can find a natural number $n_{0}(\epsilon)$ with

$$
h\left(z, \sum_{j=1}^{n} k_{j} \cdot x_{j}\right) \leq \frac{\epsilon}{2}
$$

for $n \geqslant n_{0}$. Since $M$ is dense in $\mathbb{R}$, there exists some $m_{j} \in M$ such that

$$
\left|k_{j}-m_{j}\right|\left\|x_{j}\right\|<\frac{\epsilon}{2 n}
$$


for $1 \leq j \leq n$. Let us take an element $l$ from $F_{y}$ such that $l=\sum_{i=1}^{n} m_{i}^{(l)} x_{i}$. In this case, we have $l \in E_{n} \subset E$. From Proposition 3.4, we obtain

$$
\begin{aligned}
h(z, l) & \leq h\left(z, \sum_{j=1}^{n} k_{j} \cdot x_{j}\right)+h\left(\sum_{j=1}^{n} k_{j} \cdot x_{j}, l\right) \\
& \leq \frac{\epsilon}{2}+\left\|\sum_{j=1}^{n} k_{j} \cdot x_{j}-\sum_{j=1}^{n} m_{j} \cdot x_{j}\right\| \\
& \leq \frac{\epsilon}{2}+n \frac{\epsilon}{2 n} .
\end{aligned}
$$

Then, $h(z, l) \leq \epsilon$ for $l \in E$. This shows that $E$ is dense set in $X$.

Definition 3.16. An orthonormal set $B$ in a sf-IPQLS $E$ is called orthonormal basis if there exists a unique sequence $\left\{\alpha_{n}^{(z)}\right\}$ of scalars such that has a unique representation

$$
z=\sum_{n=1}^{\infty} \alpha_{n}^{(z)} \cdot x_{n} \text { and } y=\sup \left\{z \in X_{r}: z \preceq y\right\}
$$

for every $y \in E$ and $z \in F_{y}$.

Example 3.5. Let us consider the subset $B=\{\{(0,1)\},\{(1,0)\}\} \Omega_{C}\left(\mathbb{R}^{2}\right)$ of the sf-QLS $\Omega_{C}\left(\mathbb{R}^{2}\right)$. Clearly, $B$ is an orthonormal set. For every $z \in F_{y}$, there exists a unique representation as

$$
z=\alpha_{1} \cdot\{(0,1)\}+\alpha_{2} \cdot\{(1,0)\}, \quad \alpha_{1}, \alpha_{2} \in \mathbb{R} .
$$

Also $y=\sup \left\{z \in X_{r}: z \preceq y\right\}$ for every $y \in \Omega_{C}\left(\mathbb{R}^{2}\right)$ since $\Omega_{C}\left(\mathbb{R}^{2}\right)$ is sf-QLS. Thus $B$ is orthonormal basis of $\Omega_{C}\left(\mathbb{R}^{2}\right)$.

Remark 3.2. An orthonormal set in a linear inner product space is linear independent, but this is may not be true in an IPQLS. For example, let $A=\{(0, t): 0 \leq t \leq 1\}$ and $B=\{(t, 0): 0 \leq t \leq 1\}$. The set $\{A, B\}$ is an orthonormal subset of $\Omega_{C}\left(\mathbb{R}^{2}\right)$. But, according to the Definition 1.4, this set is not ql-independent.

Theorem 3.3. Let $\left(x_{n}\right)$ be an orthonormal sequence in a Hilbert QLS $H$ and $\left(\alpha_{n}\right)$ be a sequence of real numbers. Then

$$
\left\|\sum_{n=1}^{k} \alpha_{n} \cdot x_{n}\right\|^{2} \leq \sum_{n=1}^{k}\left|\alpha_{n}\right|^{2} .
$$

Remark 3.3. Although distance of between any two elements of orthonormal sequence $\left(x_{n}\right)$ in a Hilbert space equal to $\sqrt{2}$, it is less than or equal to $\sqrt{2}$ in a Hilbert QLS.

Definition 3.17. An orthonormal sequence $\left(x_{n}\right)$ in a sf-IPQLS $X$ is said to be complete if

$$
z=\sum_{k=1}^{\infty}\left\|\left\langle z, x_{k}\right\rangle\right\|_{\Omega(\mathbb{R})} x_{k} \text { and } y=\sup F_{y}
$$

for every $y \in X$ and $z \in F_{y}$.

It is important to remember that since the right side of (3.2) is an infinite series, this equality means

$$
h_{X}\left(z, \sum_{k=1}^{n}\left\|\left\langle z, x_{k}\right\rangle\right\|_{\Omega(\mathbb{R})} x_{k}\right) \rightarrow 0 \quad(n \rightarrow \infty) .
$$


Note that a complete orthonormal sequence $\left(x_{n}\right)$ in an IPQLS $X$ is an orthonormal basis, just the same as in linear spaces. It suffices to show the uniqueness. Indeed, if $z=\sum_{k=1}^{\infty} \alpha_{k} \cdot x_{k}$ and $z=\sum_{k=1}^{\infty} \beta_{k} \cdot x_{k}$ for every $y \in X$ and $z \in F_{y}$, then

$$
0=\|z-z\|^{2}=\left\|\sum_{k=1}^{\infty} \alpha_{k} \cdot x_{k}-\sum_{k=1}^{\infty} \beta_{k} \cdot x_{k}\right\|^{2}=\left\|\sum_{k=1}^{\infty}\left(\alpha_{k}-\beta_{k}\right) \cdot x_{k}\right\|^{2}=\sum_{k=1}^{\infty}\left|\alpha_{k}-\beta_{k}\right|^{2}
$$

by Theorem 3.3 and Proposition 3.4. This means that $\alpha_{n}=\beta_{n}$ for each $n \in \mathbb{N}$.

The following two theorems give important characterizations of complete orthonormal sequences in sf-Hilbert quasilinear spaces.

Theorem 3.4. If an orthonormal sequence $\left(x_{n}\right)$ in a sf-Hilbert $Q L S H$ is complete, then $\left\langle x, x_{n}\right\rangle=$ $\{0\}$ for all $n \in \mathbb{N}$ implies $x=\theta$.

Proof. Suppose that $\left(x_{n}\right)$ is a complete orthonormal sequence in $H$. Then we have $z=$ $\sum_{k=1}^{\infty}\left\|\left\langle z, x_{k}\right\rangle\right\|_{\Omega(\mathbb{R})} x_{k}$ for any $y \in H$ and $z \in F_{y}$. Thus, if $\left\langle z, x_{k}\right\rangle=\{0\}$ for each $k \in \mathbb{N}$, then $z=\theta$.

Theorem 3.5. If an orthonormal sequence $\left(x_{n}\right)$ in a sf-Hilbert QLS $H$ is complete then

$$
\|z\|^{2} \leq \sum_{n=1}^{\infty}\left\|\left\langle z, x_{n}\right\rangle\right\|_{\Omega(\mathbb{R})}^{2}
$$

for every $y \in H$ and $z \in F_{y}$.

Proof. If the orthonormal sequence $\left(x_{n}\right)$ is complete, then for every $z \in F_{y}$ and $y \in H$

$$
z=\sum_{n=1}^{\infty}\left\|\left\langle z, x_{n}\right\rangle\right\|_{\Omega(\mathbb{R})} x_{n}
$$

Taking into account the equality

$$
\|z\|^{2}=\left\|\sum_{n=1}^{\infty}\right\|\left\langle z, x_{n}\right\rangle\left\|_{\Omega(\mathbb{R})} x_{n}\right\|^{2}
$$

and Theorem 3.3, we obtain

$$
\|z\|^{2} \leq \sum_{n=1}^{\infty}\left\|\left\langle z, x_{n}\right\rangle\right\|_{\Omega(\mathbb{R})}^{2}
$$

Definition 3.18. In an IPQLS $X$, a set $A$ is said to be complete if only the element $\theta$ is orthogonal to each element of $A$.

Example 3.6. We know from Example 2.4 that $\Omega_{C}(\mathbb{R})$ is an IPQLS with inner product defined by $\langle A, B\rangle=\{a \cdot b: a \in A, b \in B\}$ for $A, B \in \Omega_{C}(\mathbb{R})$. Since $\{0\} \in \Omega_{C}(\mathbb{R})$ is orthogonal to each elements of $\Omega_{C}(\mathbb{R}), \Omega_{C}(\mathbb{R})$ is complete. Also, the set of symmetric elements of $\Omega_{C}(\mathbb{R})$ is complete. Similarly, any symmetric subset of $\Omega_{C}(\mathbb{R})$ and the set of singular elements of $\Omega_{C}(\mathbb{R})$ are complete.

Proposition 3.5. Let $Y$ be a subspace of an IPQLS $X$ and $x \perp Y$. Then $\|x-y\| \leq\left(\|x\|^{2}+\|y\|^{2}\right)^{\frac{1}{2}}$ for every $y \in Y$. 
Proof. Firstly, since it can be written that

$$
\begin{aligned}
\langle\alpha \cdot x+\beta \cdot y, \alpha \cdot x+\beta \cdot y\rangle & \subseteq \alpha \cdot\langle\alpha \cdot x+\beta \cdot y, x\rangle+\beta \cdot\langle\alpha \cdot x+\beta \cdot y, y\rangle \\
& \subseteq \alpha^{2} \cdot\langle x, x\rangle+\alpha \beta \cdot\langle x, y\rangle+\beta \alpha \cdot\langle x, y\rangle+\beta^{2} \cdot\langle y, y\rangle
\end{aligned}
$$

we obtain,

$$
\begin{aligned}
\|x-\alpha \cdot y\|^{2} & =\|\langle x-\alpha \cdot y, x-\alpha \cdot y\rangle\|_{\Omega(\mathbb{R})} \\
& \leq\|\langle x, x\rangle\|_{\Omega(\mathbb{R})}+\|\langle x, y\rangle\|_{\Omega(\mathbb{R})}+\|\langle x, y\rangle\|_{\Omega(\mathbb{R})}+\|\langle y, y\rangle\|_{\Omega(\mathbb{R})} \\
& =\|x\|^{2}+\|\langle x, y\rangle\|_{\Omega(\mathbb{R})}+\|\langle x, y\rangle\|_{\Omega(\mathbb{R})}+\|y\|^{2}
\end{aligned}
$$

for $\alpha=1$ and $\beta=-1$. From this inequality, we write that

$$
\|x-y\|^{2} \leq\|x\|^{2}+\|y\|^{2}
$$

since $\|\langle x, y\rangle\|_{\Omega(\mathbb{R})}=0$ taking into account $x \perp Y$.

Remark 3.4. In a subspace $Y$ of a linear space $X, x \perp Y$ iff $\|x-y\| \geqslant\|x\|$ for every $y \in Y$. But, this situation may not be satisfied in a subspace of an IPQLS:

Example 3.7. Consider $X=\Omega_{C}(\mathbb{R})$ and $Y=X_{r}$. Now, if $x \in Y^{\perp}$ i.e., $x=\{0\}$, then $\|x-y\|=\|-y\| \geqslant\{0\}=\|x\|$ is provided since $Y^{\perp}=\{0\}$. But, the converse of this case may not be true. For example, let us consider the element $x=[-1,1]$ of $X$.

Let $x=[-1,1] \in X$. Since $\Omega_{C}(\mathbb{R})$ is NQLS with $\|A\|=\sup _{a \in A}|a|$, we say $\|x\|=1$. On the other hand, it can be written that $\{y\}=[y, y]$ for every $y \in Y$.

If $y \in \mathbb{R}^{+}$, then $\|x-y\|=\|[-1,1]-[y, y]\|=\|[-1-y, 1-y]\|=\sup _{y \in \mathbb{R}^{+}}|-1-y|=$ $1+y \geq 1=\|x\|$.

If $y \in \mathbb{R}^{-}$, we obtain $\|x-y\|=\|[-1,1]-[y, y]\|=\|[-1-y, 1-y]\|=\sup _{y \in \mathbb{R}^{-}}|1-y|=$ $1-y \geq 1=\|x\|$.

But $x \notin Y^{\perp}$.

\section{CONCLUSION}

In this paper, we examine the properties of orthogonal and orthonormal sets on IPQLS. We also give some theorems that their proofs are different from its classical linear analogue. We harp upon new concepts such as complete orthonormal sequence and basis in quasilinear spaces.

\section{COMPETING INTEREST}

The authors declare that they have no conflict of interests.

\section{Authors' CONTRIBUtions}

The authors declare that the work was realized in collaboration with the same responsibility. All authors read and approved the final version of this paper.

Acknowledgements. The authors would like to express their gratitude to the anonymous referees for their helpful comments and suggestions.

\section{REFERENCES}

[1] Aseev, S. M., Quasilinear operators and their application in the theory of multivalued mappings, Proc. Steklov Inst. Math., 2 (1986), 23-52

[2] Alefeld, G. and Mayer, G., Interval Analysis: Theory and Applications, J. Comput. Appl. Math., 121 (2000), 421-464

[3] Banazılı, H. K., On Quasilinear Operators Between Quasilinear Spaces, Ms. C. Thesis, İnönü University, Malatya, 2014 
[4] Çakan, S., Some New Results Related to Theory of Normed Quasilinear Spaces, Ph.D. Thesis, İnönü University, Malatya, 2016

[5] Çakan, S. and Yılmaz, Y., Normed Proper Quasilinear Spaces, J. Nonlinear Sci. Appl., 8 (2015), 816-836

[6] Lakshmikantham, V., Gnana Bhaskar, T. and Vasundhara Devi, J., Theory of set differential equations in metric spaces, Cambridge Scientific Publishers, Cambridge, 2006

[7] Rojas-Medar, M. A., Jiménez-Gamerob, M. D., Chalco-Canoa, Y. and Viera-Brandão, A. J., Fuzzy quasilinear spaces and applications, Fuzzy Sets and Systems, 152 (2005), $173-190$

[8] Yılmaz, Y., Çakan, S. and Aytekin, Ş., Topological quasilinear spaces, Abstr. Appl. Anal., (2012), Article ID 951374, 10 pp.

\section{${ }^{1}$ Department of Mathematics}

BATMAN UNIVERSITY

72100, BATMAN, TURKEY

Email address: hacerbozkurt86@gmail.com

${ }^{2}$ Department of Mathematics

İNÖNÜ UNIVERSITY

44280, MALATYA, TURKEY

Email address: yyil maz44@gmail.com

Email address: sumeyye.tay@gmail.com 